\title{
Cervical spine surgery in professional athletes: a systematic review
}

\author{
Andrei F. Joaquim, MD, PhD, ${ }^{1}$ Wellington K. Hsu, MD, ${ }^{2}$ and Alpesh A. Patel, MD² \\ 'Department of Neurology, University of Campinas (UNICAMP), Campinas, São Paulo, Brazil; and 'Department of Orthopaedic \\ Surgery, Northwestern University Feinberg School of Medicine, Chicago, Illinois
}

\begin{abstract}
Cervical surgery is one of the most common surgical spinal procedures performed around the world. The authors performed a systematic review of the literature reporting the outcomes of cervical spine surgery in high-level athletes in order to better understand the nuances of cervical spine pathology in this population.
\end{abstract}

A search of the MEDLINE database using the search terms "cervical spine" AND "surgery" AND "athletes" yielded 54 abstracts. After exclusion of publications that did not meet the criteria for inclusion, a total of 8 papers reporting the outcome of cervical spine surgery in professional or elite athletes treated for symptoms secondary to cervical spine pathology (focusing in degenerative conditions) remained for analysis. Five of these involved the management of cervical disc herniation, 3 were specifically about traumatic neurapraxia.

The majority of the patients included in this review were American football players. Anterior cervical discectomy and fusion (ACDF) was commonly performed in high-level athletes for the treatment of cervical disc herniation. Most of the studies suggested that return to play is safe for athletes who are asymptomatic after ACDF for cervical radiculopathy due to disc herniation. Surgical treatment may provide a higher rate of return to play for these athletes than nonsurgical treatment. Return to play after cervical spinal cord contusion may be possible in asymptomatic patients. Cervical cord signal changes on MRI may not be an absolute contraindication for return to play in neurologically intact patients, according to some authors. Cervical contusions secondary to cervical stenosis may be associated with a worse outcome and a higher recurrence rate than those those secondary to disc herniation. The evidence is low (Level IV) and individualized treatment must be recommended.

http://thejns.org/doi/abs/10.3171/2016.1.FOCUS15560

KEY WORDS cervical; surgery; athletes; outcomes

$\mathrm{P}$ ROPER treatment of spine injuries in high-level athletes is of paramount importance to ensure player safety as well as avoid problems with medicolegal issues. ${ }^{8,9}$ High-level athletes involved in collision sports, such as rugby, football, and wrestling, are at a higher risk of spinal cord injuries than athletes involved in other sports. ${ }^{2,3,6}$ There may be a substantial additional increase in risk for athletes with a spinal condition such as cervical spondylosis or congenital cervical stenosis.

The nuances involved in the management of athletes with cervical spine pathology are important not only because of the clinical symptoms but also for the potential impact on the patients' athletic careers. ${ }^{8}$ The outcome of anterior cervical discectomy and fusion (ACDF) for 1-level disease in the general population can be good to excellent in up to $90 \%$ of patients. ${ }^{4}$ When extrapolating data from the general population to high-level athletes, however, spine surgeons must consider different aspects of their management: the athlete may be extremely healthy, in general, but may engage in an activity with a high level of physical demands that may put his spine at risk for new injury. The spines of high-performance athletes are under unique forces of compression and repetitive and intensive shear, increasing the prevalence of spondylosis., ${ }^{1,8,9}$ Additionally, spine problems may affect the athlete's psychological state, which may lead to early retirement. ${ }^{8,9}$

Considering the unique aspects involved in the management spinal diseases of professional athletes, we performed a systematic review of papers reporting the outcomes of cervical spine surgery in high-level athletes. 


\section{Methods}

We performed a systematic literature search using the MEDLINE database up to and including August 16, 2015, using the terms "cervical spine" AND "surgery" AND "athletes." A total of 54 articles were obtained. We also screened for cross-referenced articles by manually searching the key references. In order to be included, papers had to report the outcome of cervical spine surgery in professional or elite athletes treated for symptoms due to cervical spine pathology (cervical disc herniation, spinal cord contusions due to spinal stenosis, and degenerative conditions of the cervical spine). We excluded papers that focused exclusively on nonprofessional athletes or military personnel, studies involving patients with cervical fractures and/ or dislocations, studies in which patients did not undergo cervical spine surgery, and papers without any reported outcome measure after a surgical intervention.

Data pertaining to the following outcome measures were retrieved when reported: relief of preoperative symptoms, return to play, retirement, postoperative performance, career length after surgery, and permanent neurological deficits. After all 3 authors had reviewed each of the articles, a total of 8 papers met the criteria for inclusion.

\section{Study Quality Assessment}

Studies were classified according to their level of evidence using the criteria adopted by the North American Spine Society (Levels of Evidence for Primary Research Question as Adopted by the North American Spine Society January 2005, accessible at https://www.spine.org/ Documents/ResearchClinicalCare/LevelsOfEvidence. pdf), adapted from the Oxford Centre for Evidence-Based Medicine (OCEBM) Levels of Evidence (http://www. cebm.net/ocebm-levels-of-evidence/).

\section{Results}

A total of 174 cervical cases were reported in the 8 articles. ${ }^{5,8-11,13,15}$ One hundred thirty-four patients (77\%) were football players, while 44 athletes were from other sports (baseball, rugby, wrestling, basketball). Six studies included football players, and 3 of these included only football players. ${ }^{5,8-10,15}$ One study included only baseball players and reported 11 cases of cervical disc disease, and another included only rugby players and reported 19 cases. ${ }^{1,13}$ All articles represented either Level III or Level IV evidence. A flowchart is presented illustrating our search process and inclusion/exclusion criteria (Fig. 1).

After analyzing the studies on an individual basis, we grouped them according to the primary diagnosis or hypothesis: Group 1, cervical disc herniation (5 studies, summarized in Table 1); $1,8,9,11,13$ and Group 2, traumatic neurapraxia, also described as transient spinal cord injury (3 studies, summarized in Table 2). 5,10,15

\section{Cervical Disc Herniation}

Meredith et al. ${ }^{11}$ reported a case series describing the outcomes of cervical disc herniation in 16 National Football League (NFL) athletes from the United States. The authors reviewed a single team's records for the period from 2000 to 2011 in order to identify patients with MRIdocumented disc herniation. In $81 \%$ of the cases (13 of 16 cases), the most common athlete positions represented were: lineman, linebacker, and defensive back. A single traumatic event resulting in cervical radiculopathy was the most common form of clinical presentation (9 [56\%] of 16 cases). Transient paresis was reported in 3 athletes. Anterior cervical discectomy and fusion (ACDF) was performed in 3 athletes (18.7\%) after failure of nonoperative treatment, but only 1 returned to play; all 3 athletes had spinal cord signal change on MRI. Five players were
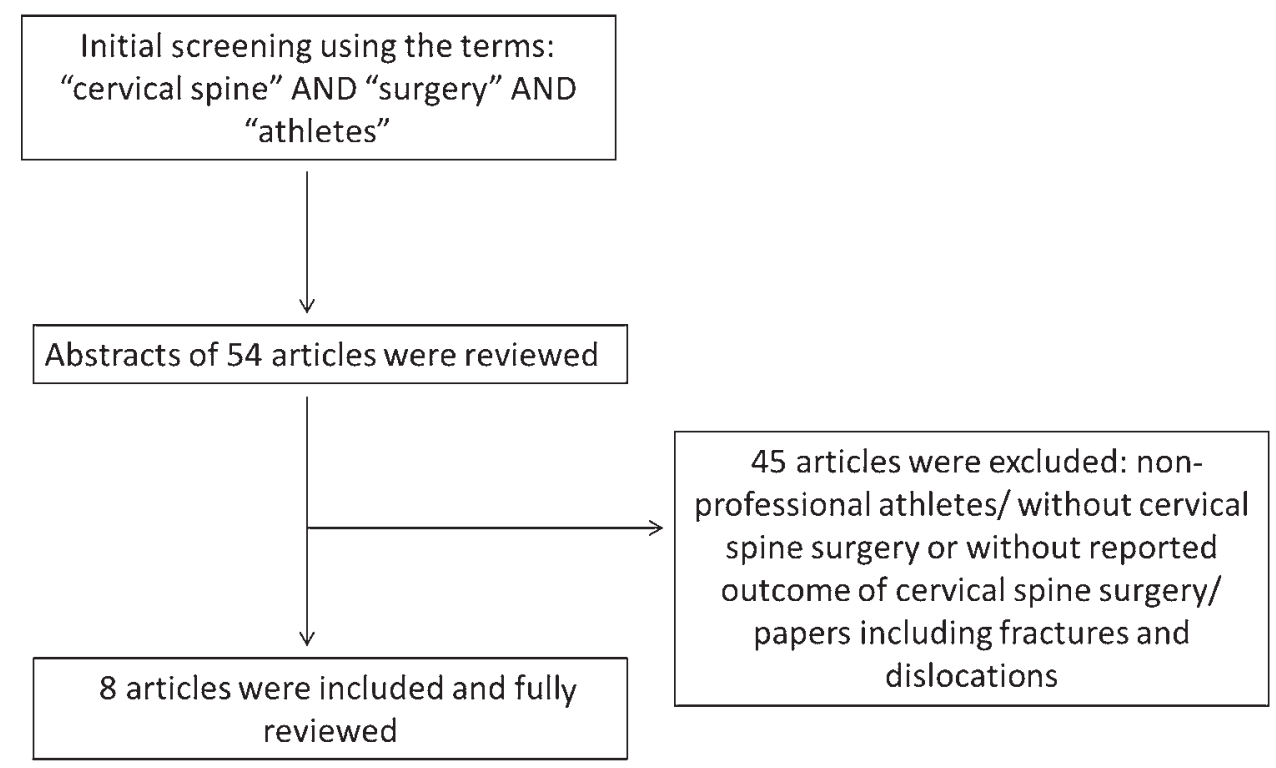

FIG. 1. Flowchart showing the selection process for the papers included in our review, according to our search methodology and inclusion/exclusion criteria. 


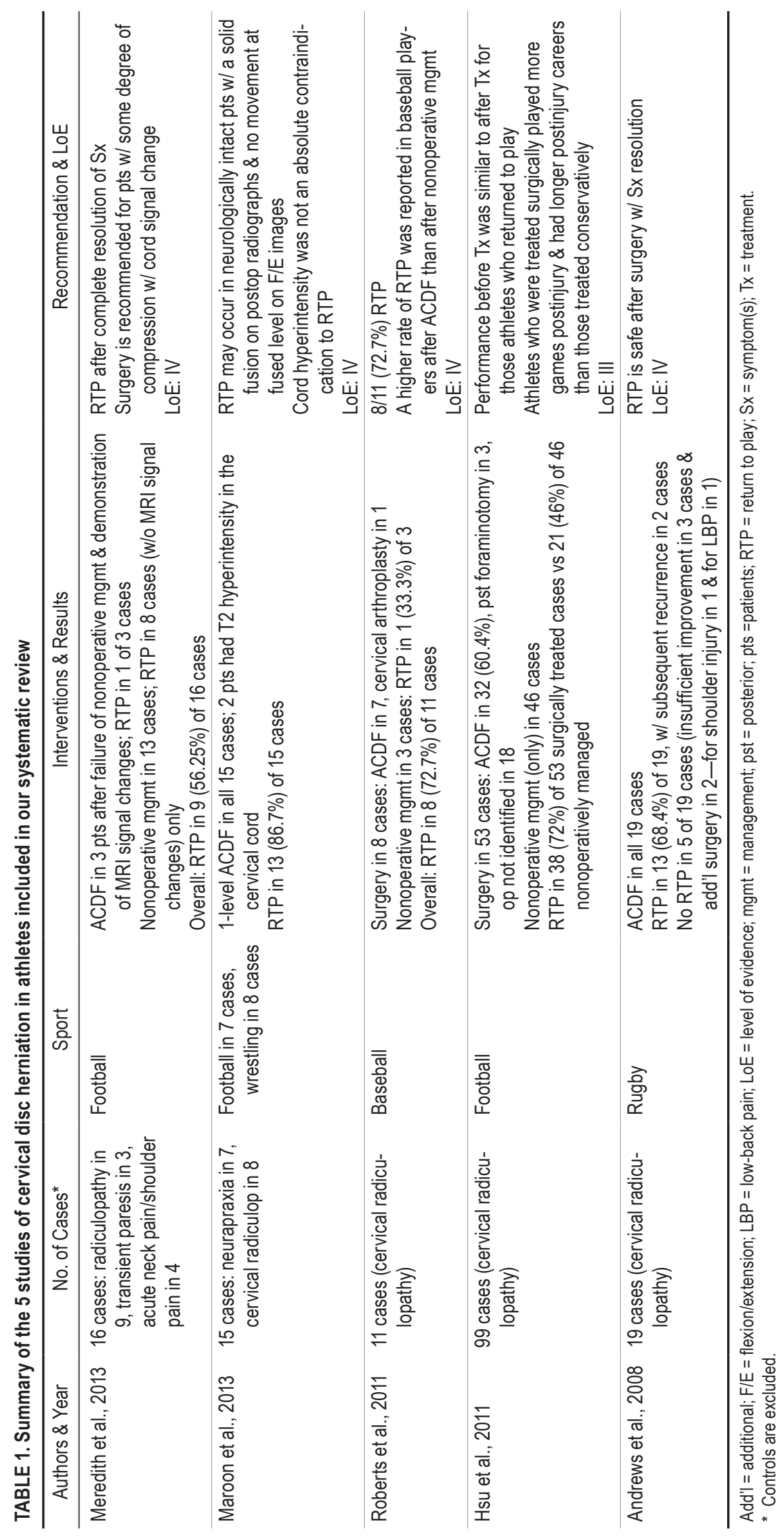




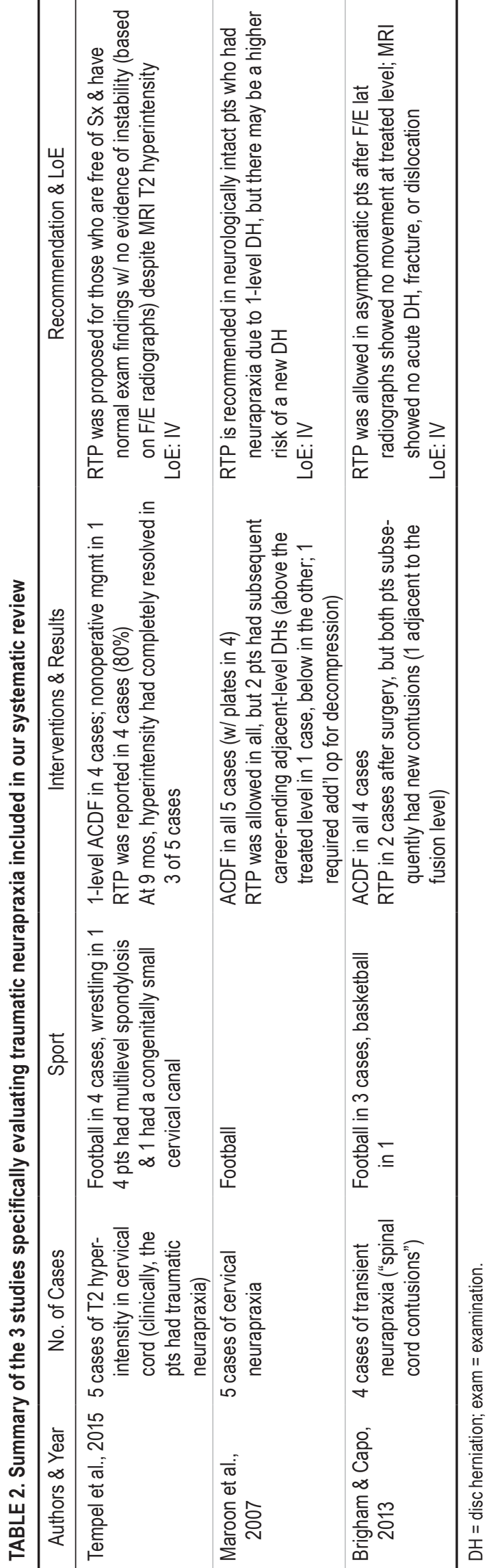

treated nonoperatively and did not return to play: 2 of these players had cord compression and spinal cord signal change but retired rather than undergo surgical treatment, and the other 3 players were cleared for return to play but were released by the team. Eight players $(50 \%)$ were treated nonoperatively and returned to play. Three of these athletes had normal findings on physical examination with MRI demonstrating disc abutment to the spinal cord but without spinal cord signal change, and all 3 returned to sport after resolution of their clinical symptoms. The remaining 5 athletes had root compression and were treated symptomatically. With a minimum of 1 year's follow-up, there was no subsequent traumatic spinal cord injury.

The authors propose that NFL athletes with cervical disc herniation can return to play after treatment, suggesting that athletes with some degree of spinal cord compression with cord signal change should receive surgical treatment, while those with discs abutting the cord (without cord signal change) or isolated nerve compression can be treated nonsurgically and return to play after complete resolution of symptoms. Of the 16 athletes with cervical disc herniation in this series, however, only $9(56.25 \%)$ returned to play ( 1 after an ACDF and 8 after nonoperative treatment). Level of evidence: IV (case series).

Maroon et al. ${ }^{9}$ evaluated the outcomes of ACDF performed in 15 professional athletes (7 NFL players and 8 professional wrestlers) by a single surgeon from 2003 to 2012. The return-to-play decisions were also reported. According to clinical presentation, 7 (46.7\%) of the 15 athletes had neurapraxia (transient myelopathy) and $8(53.3 \%)$ had symptoms of cervical radiculopathy, and in 2 cases (13.3\%) MRI showed T2 signal hyperintensity in the spinal cord. Cervical stenosis was noted in 14 patients. In all football players, the mechanism of injury was axial loading (flexion or extension) while blocking or tackling with the head.

The operative level was $\mathrm{C} 3-4$ in 4 cases, $\mathrm{C} 4-5$ in 1 , C5-6 in 8, and C6-7 in 2. One athlete who had a C4-5 ACDF also had a subsequent C5-6 ACDF for a second injury. Return to play was allowed in all athletes after normal findings on neurological examination and radiological confirmation of early fusion. Of the 15 players, 13 (86.7\%) returned to play between 2 and 12 months after surgery (with a mean of 6 months). Five athletes did not return to play and retired after a professional career ranging from 1 to 3 years. Finally, none of the athletes had any signs or symptoms of myelopathy or radiculopathy during the study follow-up.

The authors concluded that athletes may return to contact sports after ACDF if they are neurologically intact and asymptomatic and a solid fusion on documented on radiological examination, with a bony bridging at the vertebral body interface and no movement on dynamic flexion/ extension studies. They also proposed that cervical spine cord signal changes on MRI may not be a contraindication for return to play. Level of evidence: IV (case series).

Roberts et al. ${ }^{13}$ reported the outcome of cervical disc herniation in Major League Baseball (MLB) pitchers, considering the effects on their career and athletic performance; the study included only pitchers. Forty MLB pitchers from 1984 to 2009 with a cervical or lumbar disc 
herniation were identified and included in the study. Cervical disc herniation was identified in 11 pitchers, 8 of whom were treated operatively. Surgical treatment included 7 ACDF and 1 cervical disc replacement. The pitchers' mean age $( \pm \mathrm{SD})$ at the time of the diagnosis was $30.7 \pm$ 3.7 years. Of the 3 athletes treated nonsurgically, only 1 $(33 \%)$ returned to play $(\mathrm{p}=0.15)$. The authors calculated the average career length prior to treatment versus after treatment for cervical disc herniation: 160 games over 5.1 years versus 63 games over 3.7 years, respectively. The difference was not statistically significant.

The majority of pitchers with cervical disc herniation (8 [72.7\%] of 11) returned to play (mean length of time until return to play $11.6 \pm 9.9$ months). With regard to athletic performance, pitchers with cervical disc herniation pitched significantly fewer innings per season after treatment than before treatment (mean $41.7 \pm 43.1$ vs $68.5 \pm$ 44.7 , respectively; $p=0.04$ ). For other performance measures, including earned run average before and after treatment or walks plus hits per innings pitched, no significant differences were noted in preoperative versus postoperative performance for those treated surgically. The authors concluded that baseball players with cervical disc herniation can have high rates of return to play and stable performance after treatment, even when surgery is necessary. Level of evidence: IV (case series).

$\mathrm{Hsu}^{8}$ performed a retrospective cohort study of outcomes in 99 elite athletes from the NFL after treatment of cervical disc herniation. Team injury reports were reviewed and a "performance score" was calculated on the basis of statistical data before and after diagnosis for those who had at least 2 years of follow-up. The performance score was estimated for each position based on pertinent statistical data.

Of the 99 athletes, 53 players were treated surgically. Of these surgically treated players, 32 had an ACDF (60.4\%) and 3 had a posterior foraminotomy (5.7\%); in 18 cases, the type of surgery was indeterminate from the source of data available. Thirty eight (72\%) of the surgically treated players returned to play compared with $21(46 \%)$ of the 46 players who were treated nonoperatively $(\mathrm{p}<0.04)$. In addition to returning at a higher rate, the surgically treated athletes played more games after treatment (29.3 vs 14.7 games) and also had longer post-treatment playing careers (mean 2.8 vs 1.5 years) than those treated nonoperatively $(\mathrm{p}<0.04)$.

The mean duration of follow-up from the time of injury for all the NFL players in the study was 10.4 years. There was no statistically significant difference between the treatment cohorts with respect to age at diagnosis or NFL experience. There was also no statistically significant difference between the pre- and post-treatment performance scores for either cohort. Interestingly, defensive backs, as a group, had a poorer outcome than athletes in other positions, playing in only an average of 10 games over a mean of 1.2 years period $(\mathrm{p}<0.0008)$. Finally, age at the time of diagnosis also had a negative impact on career longevity after treatment.

The author concluded that players treated surgically have higher return to play rates and greater career longevity than those treated nonoperatively. For those athletes who were able to return to play, there was no significant difference between pre- and posttreatment performance measures. Age at the time of the diagnosis of and defensive back position had a negative effect on final outcome of cervical disc herniation. Level of evidence: III (retrospective cohort).

Andrews et al. ${ }^{1}$ performed a retrospective study of the outcome ACDF for 19 professional rugby players; all of the players underwent ACDF between 1998 and 2003 for radicular pain refractory to conservative treatment. Medical records and telephone interviews were performed to better understand the clinical outcome, including return to play and long-term consequences.

The players' mean age was 28 years (range 22 to 37 years). The authors reported that neck and radicular pain improved in 17 patients (89.4\%; eradicated in 15 and partially improved in 2). Two patients $(11.7 \%$ ) had no change in their radicular pain. Considering player position, 13 were front-row forwards, 1 was a second-row forward, 2 were back-row forwards, and 3 were backs.

A total of $13(68.4 \%)$ of 19 patients returned to play, 9 of them by 6 months after surgery. One returned to professional rugby but played in a lower division, and another took more than 12 months to return to play due to concomitant back pain problems. Five players did not return to play: 3 due to insufficient improvement in the symptoms ( 2 of them had persistent neck pain requiring additional surgeries without improvement and 1 had a triceps weakness), 1 due to an additional shoulder injury requiring surgery, despite improvement in the cervical problem, and the last due to persistent low-back pain, despite improvement in his cervical symptoms.

Of the 13 players who returned to play, 2 had a recurrence of symptoms. In one of those 2 players, the recurrence was due to adjacent-level disease and did not preclude him from play; the other player retired 2 years postoperatively due to neck pain. The majority of the players with problems in the neck were front-row forwards. The authors concluded that return to playing rugby is both likely and safe for players who have undergone ACDF. Level of evidence: IV (case series).

\section{Traumatic Neurapraxia or Transient Spinal Cord Injury}

Three of the reviewed studies reported on 14 patients with traumatic neurapraxia associated with professional sports; the patients included 12 professional football players, 1 basketball player, and 1 professional wrestler.

Tempel et al..$^{15}$ retrospectively reported the outcome of 5 cases involving professional athletes (4 NFL players and 1 professional wrestler) who had traumatic neuropraxia that had resolved at the time of medical consultation. All of the athletes had a MRI T2 signal hyperintensity in the cervical spinal cord: in 4 cases it was due to multilevel spondylosis, and in 1 case it was due to a congenital small cervical canal. In 4 cases, the neurapraxia was due to compression at the $\mathrm{C}-4$ level; in 1 case it was due to compression at the C5-6 level; and in 1 case it was due to compression by the C- 4 vertebral body. Four cases were managed surgically with a single-level ACDF; in the remaining case, the athlete received only nonsurgical treatment. MRI was performed 3 months after injury in each 
case. In 4 cases, the study showed partial resolution of T2 signal hyperintensity; in the remaining case, the signal hyperintensity was unchanged. Four players were allowed to return to participation in sports, but only $2(40 \%)$ of them returned to professional sport without any additional episodes of neurapraxia or related cervical spine conditions. The authors concluded that athletes who undergo surgical treatment and are asymptomatic postoperatively, without evidence of spinal stability (based on flexion/extension radiographs), are able to return to sport even in the setting of T2 signal hyperintensity and a previous episode of traumatic neurapraxia. Level of evidence: IV (case series).

Maroon et al. ${ }^{10}$ reported on 5 elite football athletes with cervical neurapraxia (transient posttraumatic loss of motor and/or sensory function due to loss of conductance in the cervical spinal cord after trauma). The athletes' ages ranged from 20 to 32 years (mean of 27.2 years) at the time of injury. All of them had bilateral paresthesias (involving all 4 extremities in 3 cases and only the upper limbs in 2), lasting from a few minutes to more than 24 hours. Two athletes had transient motor deficits, but without permanent sequelae. All of them had herniated discs with focal spinal cord compression but without parenchymal changes. All 5 underwent an ACDF, with cervical plates in 4 cases. All the players were allowed to return to active play after rehabilitation; the timing of their return to play ranged from 9 weeks to 8 months after surgery. Of note, 2 of the 5 players subsequently had adjacent-level disc herniation, which ended their careers in both cases and required a second operation in one. The adjacent-level disease was at the level inferior to the treated level in one case and superior to it in the other.

The authors concluded that neurologically intact athletes who have had cervical neurapraxia due to focal cord compression and a single-level herniation and have undergone surgical decompression and fusion may return to play when they are asymptomatic and imaging studies demonstrate fusion. Level of evidence: IV (case series).

In a retrospective study, Brigham and Capo reported the long-term follow up (minimum of 2 years after injury) of 4 athletes ( 3 football players and 1 basketball player) who had cervical spinal cord contusions with transient deficits. ${ }^{5}$ None of them had an acute disc herniation, fracture, spinal instability, or focal cord compression. Outcomes and return to play were reported, as well as the correlation of MRI findings with clinical signs and symptoms. All of the athletes had congenital cervical stenosis (with a lack of CSF signal around the spinal cord on an axial MR image). All underwent anterior cervical fusions at the index level of their contusion (at C3-4 in 3 cases and C5-6 in 1 case) and returned to play. Although none of the players had permanent neurological deficits, 2 (50\%) suffered new contusions -5 years later and adjacent to the level fused in one case (C3-4 fused after initial injury; subsequent injury at the C4-5 level, treated nonsurgically) and 2 years later and not at a level adjacent to the previously fused level in the other case (C5-6 fused after initial injury; subsequent injury at C3-4, also treated with ACDF). The mechanism of injury was cervical spine hyperextension. The presence of a contusion did not correlate with athletes' symptoms and signs.
The authors postulated that the horizontal facet orientation of the C3-4 level associated with congenital stenosis and cervical hyperextension may be responsible for cervical spinal cord contusion in athletes. ACDF at C3-4 seems important to preventing clinical deterioration in these cases. Decisions regarding return to play in this scenario may be based on the athletes' clinical condition, as there was a poor correlation between the MRI findings and clinical presentation. Finally, this group of patients with congenital stenosis may have a high rate of symptom recurrence, and the long-term consequences of the cumulative cervical cord trauma are not known. Level of evidence: IV (case series).

\section{Discussion}

We report the results of 8 studies investigating cervical spine conditions and treatment in athletes. The majority of the studies included football players..$^{5,8-11,15}$ Other contact sports (including baseball, rugby, wrestling, and basketball) were represented by only 40 of the 174 patients included in our review. Caution must therefore be taken before extrapolating the results of our analysis to other sports, such as soccer, gymnastics, or swimming, which are underrepresented in or absent from the literature.

\section{Cervical Disc Herniation}

The 5 studies specifically focusing on cervical disc herniation reported on cases involving a total of 98 initial surgeries. In 18 of these 98 cases, the type of surgery was not identified. Of the remaining 80 procedures, 77 were anterior cervical operations. Single-level ACDF was performed in 76 (95\%) of 80 cases. Posterior foraminotomy was performed in 3 cases $(3.75 \%)$, and cervical arthroplasty in 1 case $(1.25 \%)$. These data suggest that in highlevel athletes, treating surgeons often perform an anterior procedure, potentially avoiding muscular and ligamentous injuries associated with posterior cervical approaches. Additionally, fusion is preferred over cervical arthroplasty, probably because of the better-studied long-term durability of the procedure. ${ }^{1,8,11,13}$ In all of the studies of cervical disc herniation, more than $50 \%$ of players were able to return to play. However, the largest study (Hsu's study of 99 NFL athletes with herniated discs) ${ }^{8}$ showed a return-to-play rate of only $46 \%$ for athletes whose injuries were managed nonsurgically, as opposed to a rate of $72 \%$ in the group treated surgically. The study by Maroon et al. ${ }^{10}$ shows a particularly high return-to-play rate of $86.7 \%$ (13 of 15 cases). This high rate may be explained by the fact that these authors proposed that cervical spinal cord signal changes are not a contraindication for return to play. Although return to play after ACDF is acceptable in most of the current sports guidelines articles, these data refer to discectomy for treating cervical radiculopathy and may not be valid for cervical myelopathy.,12

\section{Traumatic Neurapraxia or Transient Spinal Cord Injury}

Tempel et al. ${ }^{15}$ reported on 5 patients who had MRI T2 signal hyperintensity in the cervical spinal cord and after experiencing traumatic neurapraxia. Despite the signal change, all 5 patients were asymptomatic by the time 
of medical consultation. Only $2(20 \%)$ of the athletes returned to sport, however. Both had documented spinal fusion and normal findings on clinical examination, but they did have persistent T2 hyperintensity on MRI as well as the history of traumatic neurapraxia. On the other hand, in the study by Maroon et al. ${ }^{10}$ which also involved 5 athletes with transient neurapraxia, all 5 athletes had herniated discs with focal cord compression but did not have any evidence of parenchymal changes, and they all returned to play after ACDF. Although 2 players each had a subsequent additional disc herniation, which ended their careers (one in the 7th and the other in 27th game after surgery-both tackling), the return to play rate in this study $(100 \%)$ was much higher than in the study by Tempel et al., ${ }^{15}$ suggesting that different clinical and radiological scenarios are involved in spinal cord injury. Bailes proposed that surgeons must advise athletes in contact sports that neurapraxia may be associated with an increased risk of permanent spinal cord injury. ${ }^{2}$ Additionally, patients with multiple transient or permanent neurological deficits may not return to sport. ${ }^{3}$ The recommendation of return to play after a neurological examination with normal findings, a spinal cord decompression, and a segmental fusion must be balanced with education as to the risk of permanent spinal cord injury or a new compression at an adjacent level. Individualizing the recommendation to return to sport is important, taking into consideration the athlete's neurological status and neuroimaging findings as well as social and financial factors.

Schroeder et al..$^{14}$ evaluated the impact of preexisting cervical spine pathology on the careers of NFL athletes. Athletes who had a preexisting cervical spine diagnosis (143) were compared with matched controls. The authors reported that although cervical spine pathology may affect the careers of athletes, the performance-based outcomes were not unfavorable in those with preexisting cervical pathology, even when surgery was required. However, the careers of those athletes with cervical pathology were shorter than the careers of those without it. The only condition associated with a worse performance and also a short career was cervical spondylosis. A potential explanation for that is that spondylosis is a progressive disease that may worsen with constant trauma in professional football. Seven of the 9 athletes who had had cervical spine surgery were drafted (one also had signal change in cervical MRI), and all of them were able to play. These data emphasize the fact that cervical surgery is not an absolute contraindication for playing contact sports.

Finally, cervical cord contusions associated with congenital cervical stenosis, but without acute disc herniation, fractures, or evident instability, were associated with a high rate of recurrence. ${ }^{5}$ Of 4 patients with this condition, 2 had a new injury even after ACDF at the index level. Associated with hyperextension, this condition may permit return to play when patients are asymptomatic, but the possibility of a devastating and catastrophic injury cannot be neglected. ${ }^{2,3,5}$ The higher rate of new contusions in the published series $(50 \%)$ may be considered as a negative factor weighing against release of these athletes to contact sports after this type of injury. Additionally, spine surgeons must differentiate a cervical cord contusion sec- ondary to a disc herniation, which may have a more favorable prognosis,${ }^{14}$ from a contusion secondary to congenital cervical stenosis. ${ }^{5}$ Torg et al. ${ }^{16}$ proposed that those patients with symptoms lasting longer than 36 hours, multiple episodes of spinal cord concussion, or evidence of edema on MRI should avoid return to play (absolute contraindications to return to sports). Although we found studies that suggest that return to play in asymptomatic patients who have a T2 signal change in the cervical spinal cord may be reasonable, the data on the long-term outcome of these patients are not known, and they may have a heightened risk of neurological sequelae.

Our review is limited by the low level of evidence of the retrieved studies and their retrospective nature. Although 2 papers provided retrospective comparisons of surgical and nonsurgical management, the literature is insufficient to provide a strong recommendation for treatment. Weak recommendations for the surgical management of cervical disc herniation, transient neurapraxia, and congenital stenosis with cord signal change can be made. The studies did not investigate the outcomes of different return-to-play criteria. Therefore no recommendations can be made for a specific return-to-play protocol that would predict safety, recurrence, or performance. Given the consensus in the literature, a strong recommendation can be made that athletes should have undergone surgical decompression and successful stabilization as well as have complete resolution of symptoms prior to considering return to play. Lastly, the available literature only focuses on male athletes, and the results may not be applicable to female high-level athletes.

\section{Conclusions}

The majority of the patients included in this review were football players. ACDF is the surgery most commonly performed for the treatment of cervical spine injuries in high-level athletes. Most of the studies suggest that return to play after ACDF is safe for athletes who were treated for cervical radiculopathy secondary to disc herniation. Surgical treatment may provide a higher return to play in these athletes than nonsurgical treatment.

Return to play after cervical spine contusion may be possible in asymptomatic patients. Some authors proposed that even neurapraxia, cervical spinal cord signal changes on MRI, or both may not be an absolute contraindication for return to play in neurologically intact patients. The evidence for this is low, and individualized treatment must be recommended. Cervical contusions secondary to cervical stenosis may have a worse outcome and a higher recurrence rate than those secondary to disc herniation.

\section{References}

1. Andrews J, Jones A, Davies PR, Howes J, Ahuja S: Is return to professional rugby union likely after anterior cervical spinal surgery? J Bone Joint Surg Br 90:619-621, 2008

2. Bailes JE: Experience with cervical stenosis and temporary paralysis in athletes. J Neurosurg Spine 2:11-16, 2005

3. Bailes JE, Hadley MN, Quigley MR, Sonntag VK, Cerullo LJ: Management of athletic injuries of the cervical spine and spinal cord. Neurosurgery 29:491-497, 1991

4. Bohlman HH, Emery SE, Goodfellow DB, Jones PK: Robinson anterior cervical discectomy and arthrodesis for cervi- 
cal radiculopathy. Long-term follow-up of one hundred and twenty-two patients. J Bone Joint Surg Am 75:1298-1307, 1993

5. Brigham CD, Capo J: Cervical spinal cord contusion in professional athletes: a case series with implications for return to play. Spine (Phila Pa 1976) 38:315-323, 2013

6. Cantu RC, Mueller FO: Catastrophic spine injuries in football (1977-1989). J Spinal Disord 3:227-231, 1990

7. Dailey A, Harrop JS, France JC: High-energy contact sports and cervical spine neuropraxia injuries: what are the criteria for return to participation? Spine (Phila Pa 1976) 35 (21 Suppl):S193-S201, 2010

8. Hsu WK: Outcomes following nonoperative and operative treatment for cervical disc herniations in National Football League athletes. Spine (Phila Pa 1976) 36:800-805, 2011

9. Maroon JC, Bost JW, Petraglia AL, Lepere DB, Norwig J, Amann C, et al: Outcomes after anterior cervical discectomy and fusion in professional athletes. Neurosurgery 73:103112,2013

10. Maroon JC, El-Kadi H, Abla AA, Wecht DA, Bost J, Norwig $\mathrm{J}$, et al: Cervical neurapraxia in elite athletes: evaluation and surgical treatment. Report of five cases. J Neurosurg Spine 6:356-363, 2007

11. Meredith DS, Jones KJ, Barnes R, Rodeo SA, Cammisa FP, Warren RF: Operative and nonoperative treatment of cervical disc herniation in National Football League athletes. Am J Sports Med 41:2054-2058, 2013

12. Morganti C, Sweeney CA, Albanese SA, Burak C, Hosea T, Connolly PJ: Return to play after cervical spine injury. Spine (Phila Pa 1976) 26:1131-1136, 2001

13. Roberts DW, Roc GJ, Hsu WK: Outcomes of cervical and lumbar disk herniations in Major League Baseball pitchers. Orthopedics 34:602-609, 2011
14. Schroeder GD, Lynch TS, Gibbs DB, Chow I, LaBelle MW, Patel AA, et al: The impact of a cervical spine diagnosis on the careers of National Football League athletes. Spine (Phila Pa 1976) 39:947-952, 2014

15. Tempel ZJ, Bost JW, Norwig JA, Maroon JC: Significance of T2 hyperintensity on magnetic resonance imaging after cervical cord injury and return to play in professional athletes. Neurosurgery 77:23-31, 2015

16. Torg JS, Guille JT, Jaffe S: Injuries to the cervical spine in American football players. J Bone Joint Surg Am 84A:112-122, 2002

\section{Disclosures}

Dr. Patel reports direct stock ownership in Amedica, Cytonics, and Vital5; a consultant relationship with Amedica, Biomet, DePuy, Stryker, Pacira, and Relievant; and receipt of royalties from Biomet, Ulrich, and Amedica.

\section{Author Contributions}

Conception and design: all authors. Acquisition of data: all authors. Analysis and interpretation of data: all authors. Drafting the article: all authors. Critically revising the article: all authors. Reviewed submitted version of manuscript: Joaquim, Hsu. Approved the final version of the manuscript on behalf of all authors: Joaquim. Study supervision: all authors.

\section{Correspondence}

Andrei F. Joaquim, Neurosurgery Division, Department of Neurology, University of Campinas, Campinas, São Paulo 13083 888, Brazil. email: andjoaquim@yahoo.com. 